\title{
La beclometasona fue más efectiva que el salmeterol en niños pero se asoció a leve retardo de crecimiento
}

Comparison of beclomethasone, salmeterol, and placebo in children with asthma. Simons E, Dolovich J, Moote D et al,. NEJM 1997, 337 :23,1659-65.

\section{Objetivo}

Evaluar los efectos terapéuticos de la beclometasona inhalatoria y el salmeterol en el control de pacientes asmáticos leves

Diseño

Ensayo clínico randomizado, doble ciego controlado con placebo. Seguimiento de 1 año.

Lugar

Hospital de Niños de Winnipeg, Canadá.

\section{Pacientes}

Se incorporaron 214 pacientes de 6 a 14 años con asma estable (edad promedio 9.3 años). Criterios de inclusión: no haber recibido corticoides inhalatorios en los últimos 3 meses, volumen espiratorio forzado en el primer segundo (VEF1) $>$ al $70 \%$ luego de suspender por lo menos 6 horas los broncodilatadores, $10 \%$ de aumento del VEF1 a los 30 minutos de aplicarse $400 \mathrm{mg}$ de salbutamol y requerir menos de $8 \mathrm{mg}$ de metacolina para disminuir $20 \%$ el VEF1.

\section{Intervención}

Se indicó beclometasona $400 \mathrm{mg} /$ día $(\mathrm{n}=81)$, salmeterol $100 \mathrm{mg} /$ día $(\mathrm{n}=80)$ y placebo $(\mathrm{n}=80)$. Se evaluó la reactividad de la vía aérea al inicio del tratamiento y a los 3, 6, 9 y 12 meses y a las 2 semanas luego de la suspensión. Se indicó registrar diariamente los síntomas, la medicación de rescate utilizada, el ausentismo escolar y la medición del flujo pico matinal y vespertino.

\section{Medición de resultados principales}

Reactividad de la vía aérea evaluada por la dosis de metacolina necesaria para disminuir el $20 \%$ del VEF1 (PC20) a las 12 y 36 horas de haber suspendido la medicación que recibía. Resultados secundarios: parámetros espirométricos; días y noches sin medicación de rescate, tipo de medicación de rescate, días de ausencia escolar, abandono del estudio, efectos adversos y progresión de la altura del paciente.

\section{Resultados}

La reactividad bronquial disminuyó en los tres grupos, la PC20 a las $12 \mathrm{~h}$ de suspendida la medicación fue mayor al doble en el grupo beclometasona vs. los grupos salmeterol y placebo con diferencias estadísticamente significativas $(\mathrm{p}<0,001)$. Los efectos sobre la reactividad bronquial desaparecieron a las dos semanas de interrumpir el tratamiento. La reactividad de la vía aérea no varió durante el estudio en el grupo salmeterol ni tampoco al suspender el mismo. No hubo diferencias estadísticamente significativas de la PC20 a las 36 horas de suspendida la medicación entre beclometasona o salmeterol vs. placebo. Tanto la beclometasona y salmeterol mejoraron los parámetros espirométricos de los pacientes en relación a placebo $(\mathrm{p}<0.001)$. También fueron mejores los valores de flujo pico de ambas drogas no habiendo diferencias entre ambas. El grupo beclometasona tuvo mayor número de noches sin uso de medicación de rescate que el de salmeterol y el de placebo ( $92 \%$ vs. $88 \%$ vs. $83 \%$, p<0.001) y mayor número de días que no requirieron salbutamol $(p<0.03)$. El grupo beclometasona tuvo menor número de abandonos $(\mathrm{p}<0.03)$. El crecimiento promedio de altura durante el año de seguimiento fue de $3.96 \mathrm{~cm}$, $5.40 \mathrm{~cm}$ y $5.04 \mathrm{~cm}$ para los grupos beclometasona, salmeterol y placebo respectivamente. El progreso de altura del grupo beclometasona fue significativamente menor que el del placebo $(p<0.018)$ y el del salmeterol $(p<0.004)$. No hubo diferencias en el resto de indicadores secundarios.

\section{Conclusiones}

Si bien la beclometasona es más efectiva que el salmeterol para controlar a largo plazo el asma en los niños, se debería evaluar los beneficios de la misma en relación a la menor progresión de altura.

Fuente de financiamiento: Byk Gulden Foundation blema metodológico en este estudio es que la beclometasona y el salmeterol demostraron ser diferentes entre sí sólo en la hiperreactividad y en el crecimiento estatural. El resto de los resultados (síntomas, medicación de rescate, pruebas espirométricas) muestran que la beclometasona es diferente al placebo y el salmeterol no; pero no reportan diferencias significativas entre las dos drogas activas. Algo a mencionar también, es que en este trabajo la hiperreactividad no empeoró al suspender el salmeterol como en otros estudios con $\beta 2$ adrenérgicos de acción corta. Las conclusiones de estos trabajos para nuestra práctica en el manejo de los niños con asma son: a) es poco probable que una droga costosa como el cromoglicato sea efectiva en pacientes con asma moderada; y b) la utilización de la beclometasona debe considerarse sólo en pacientes con asma moderada a severa, ya que a pesar de ser efectiva retarda el crecimeinto estatural.

$$
\text { * Ver glosario }
$$

\section{Dr. Pablo Jinich}

Universidad de Medicina Familiar y Preventiva Hospital Italiano de Buenos Aires

\section{Referencias}

1. Donahue J. et. al. Inhaled steroids and the risk of hospitalization for asthma. JAMA 1997; 277: 11, 887-91

2. Cogswell J. J. Simpkiss M. J. Nebulsed sodium cromoglycate in recurrently wheezy prescho- ol children. Arch Dis Child 1985; 60: 736-38. 3. Glass J. Archer I. N. Adams W, Simpson H. Nebulised cromoglycate, teophylline, and placebo in preschool children. Arch Dis Child 1981: 56:648-51.

4. Guidelines for diagnosis and treatement asthma. Expert Panel Report. No 2 Bethesda. 1997 NIH publication $\mathrm{N}^{\circ} 97,4051$.

5. Wolthers $\mathrm{O}$ et. al. Short term growth during treatment with inhaled fluticasone propionate and beclomethasone dipropionate. Arch Dis Chil 1993; 68: 673-6.

6. Wolthers $\mathrm{O}$ et. al. Crowth of asthmatic children during treatment with budesonide: a double blind trial. BMJ 1991; 303: 163-65.

7. Allen D. Mullen B. Meta-analysis of the effect of oral and inhaled corticosteroids on growth J. Allegy Clin Inmunol 1994:93:967-976. 